\title{
Evolución del sistema de salud de Perú: buenas prácticas y desafíos en su construcción. Década 2005-2014
}

\section{Evolution of health system Peru: Good practices and challenges in construction. Decade 2005-2014}

\author{
W. Ricardo Cañizares Fuentes ${ }^{1, a}$ \\ ${ }^{1}$ Docente Universidad Católica de Santiago de Guayaquil. \\ ${ }^{a}$ Secretario Adjunto del Organismo Andino de Salud ORAS-CONHU hasta 2015.
}

\begin{abstract}
Correspondencia
Wilson Ricardo Cañizares

Fuentes

wcanizar@hotmail.com
\end{abstract}

Recibido: 14 de julio 2016.

Aceptado: 2 de noviembre 2016.

Conflictos de interés: El autor declara no tener conflictos de interés.

Financiamiento: Autofinanciado.

Citar como: Cañizares Fuentes WR. Evolución del sistema de salud de Perú: buenas prácticas y desafíos en su construcción. Década 20052014. An Fac med. 2017;78(4):44551

DOI: http://dx.doi.org/10.15381/

anales.v78i4.14269

\section{An Fac med. 2017;78(4):445-51/ http://dx.doi.org/10.15381/anales.v78i4.14269}

\section{Resumen}

Introducción: Mejorar los sistemas nacionales de salud es preocupación mundial en la actual agenda política. Los últimos años, la mayoría de países sudamericanos han hecho esfuerzos por mejorarlos introduciendo reformas y politicas públicas con el objetivo que la población tenga acceso universal a atención de salud. La Organización Mundial de la Salud recomienda estudiarlos; en ese sentido, se decidió analizar la evolución del sistema de salud del Perú en un período de 10 años con el propósito de extraer aprendizajes para fortalecerlo y también como insumo para otros países. Objetivos: Analizar la evolución, las buenas prácticas y los factores que influyen en el funcionamiento del sistema de salud de Perú. Participantes: Ex ministros y viceministros de salud, representantes de la OPS, académicos, técnicos y representantes de la sociedad civil. Métodos: Se realizó un estudio cualitativo mediante entrevistas a profundidad que fueron grabadas, transcritas y codificadas para identificar y analizar las principales representaciones sociales que tienen los entrevistados respecto al sistema de salud. Resultados: El incremento del financiamiento no significo incremento del acceso a atención de salud, la rectoría del Ministerio de Salud se fortaleció poco, falta acceso a atención integral y de calidad, se propone crear un solo fondo nacional de financiamiento y un solo plan de salud para todos los peruanos. Conclusiones: La rectoría no se ha consolidado, se reconoce el mejoramiento de la cobertura financiera pero no del acceso a servicios de salud y hay serias brechas de recursos humanos, infraestructura, equipamiento y acceso a medicamentos, a lo que se suma ineficiencia organizacional.

Palabras clave: Sistemas de salud; Políticas públicas; Financiamiento de la atención de salud.

\section{Abstract}

Introduction: The improvement of national health systems is a global concern and is part of the discussions in the current international political agenda. In the past years, most south american countries have made efforts to improve their systems introducing reforms and public policies to reach universal access to health care for all citizens. It is necessary to study them, as recommended by the World Health Organization. According to it, this present work studies the evolution of the health system in Peru over a period of 10 years aiming to extract lessons for improvement as well as to provide inputs for other countries. Objectives: To analyze the evolution, good practices and variables influencing the functioning of the health system in Peru. Participants: Former ministers and deputy ministers of health, PAHO representatives, academics, technical experts and representatives of civil society. Methods: Quality approach research, interviews that were recorded, transcribed and coded to identify and analyze the main social representations interviewers had regarding the health system. Results: Increasing funding did not result in increasing access of population to health care, the rectory of Health Ministry was slightly strengthened, and lack of access to comprehensive care and quality, interviewers are in favor of creating a single national financing fund and one health plan for all Peruvians. Conclusions: The rectory has not been strengthening enough. Financial coverage has been improved although the access to health care services has not, and there are consistent gaps in human resources, infrastructure, equipment and access to medicines, in addition to organizational inefficiency.

Keywords: Health system; Public Policies; Healthcare Financing. 


\section{INTRODUCCIÓN}

Mejorar los sistemas nacionales de salud es una preocupación mundial presente en la actual agenda política ${ }^{(1,2,3)}$. Un sistema de salud es la suma de todas las organizaciones, instituciones y recursos cuyo objetivo principal consiste en mejorar la salud ${ }^{(4)}$.También es el conjunto de relaciones políticas, económicas e institucionales responsables por la conducción de los procesos relativos a la salud de la población ${ }^{(5)}$. De igual manera, es un mecanismo político y social para la disminución de la pobreza y la inequidad $(6,7)$ al destinar financiamiento público para atender la salud especialmente de poblaciones con poco acceso a atención de salud.

En la actualidad los principales desafíos están en mejorar la equidad y alcanzar la universalidad de servicios de salud por parte de la población ${ }^{(8,9)}$, por eso la Organización Mundial de la Salud recomienda aumentar los estudios sobre los sistemas de salud incluso porque su funcionamiento trasciende al tema de salud e involucra al desarrollo social y económico de los países ${ }^{(10)}$.

La oportunidad de laborar en el Organismo Andino de salud ORAS/CONHU (11) nos permitió observar los procesos de fortalecer los sistemas de salud que están haciendo los países andinos, por lo que se consideró importante estudiar al sistema de salud del Perú como caso representativo en la región de un sistema basado en el aseguramiento universal ${ }^{(12)}$. El objetivo de este estudio fue analizar la evolución, las buenas prácticas y los factores que influyen en el funcionamiento del sistema de salud de Perú en el periodo 2005-2014.

\section{MÉTODOS}

Se diseñó un estudio con enfoque cualitativo basado en las representaciones sociales $(13,14,15)$ para explicar desde los actores sociales que han estado relacionados directa o indirectamente con la implementación y funcionamiento del sistema de salud en el Perú durante los años 2005-2014, los cambios en las 3 funciones principales de un sistema de

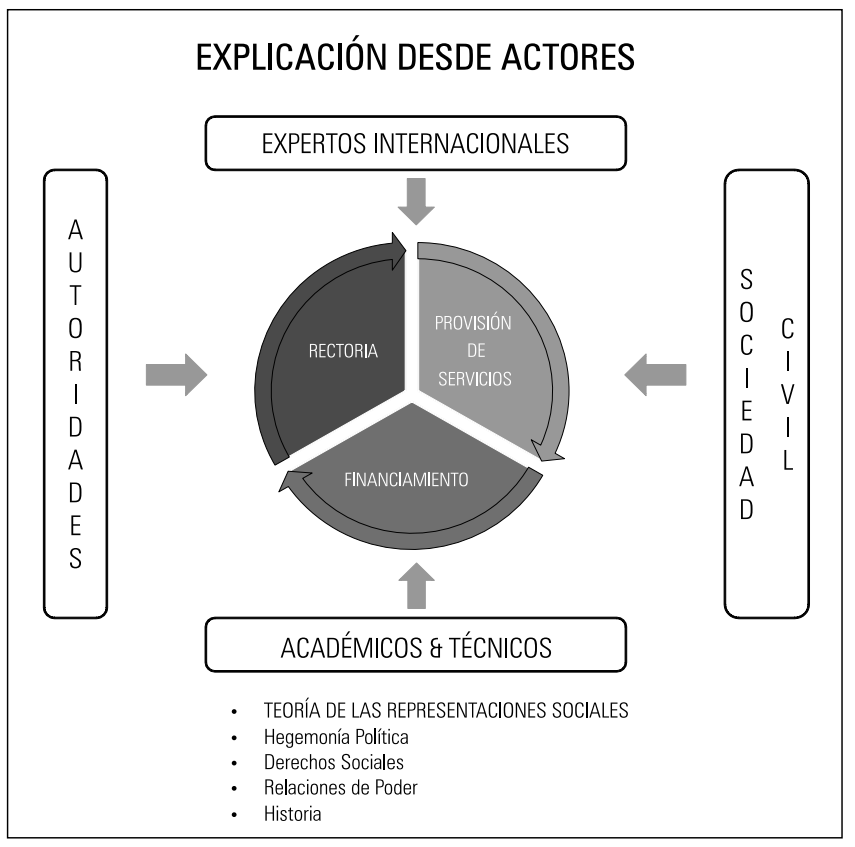

Figura 1. Enfoque cualitativo basado en las representaciones sociales: actores sociales relacionados directa o indirectamente con la implementación del sistema de salud ${ }^{(13,16)}$.

salud: rectoría, provisión de servicios y financiamiento ${ }^{(16)}$ y mediante esas explicaciones comprender los avances y los desafíos que se deben superar para mejorar. Figura 1.

La interpretación de la información se hizo en base a la teoría de las representaciones sociales de Serge Moscovici (17) la cual es una modalidad particular del conocimiento, cuya función es la elaboración de los comportamientos y la comunicación entre los individuos. Es un sistema de pensamientos que permite la relación con el mundo y con los demás, facilita interpretar el quehacer de las personas como proceso cognitivo que aportan elementos afectivos, normativos y prácticos que organizan la comunicación social e individual y constituyen una forma de expresión ${ }^{(13)}$ mediante la cual personas y grupos obtienen una lectura de la realidad y, además, toman una determinada posición en relación a ella ${ }^{(18)}$.

En ese sentido, los entrevistados analizaron y explicaron desde su visión los avances del sistema de salud peruano en los diez años estudiados y a partir de esas representaciones individuales se elaboró las representaciones colectivas que tiene este grupo respecto al hecho concreto de su sistema de salud. Se seleccionó a 13 informantes que desempeñaron cargos directivos en estos 10 años de estudio (205-2014) específicamente ministros y viceministros de salud, técnicos de salud pública, representantes de la Organización Panamericana de la Salud y de la sociedad civil así como la Academia Nacional de Medicina y Foro Salud. Se definió un periodo de 10 años considerando que es un tiempo bastante adecuado para qué se puedan observar cambios importantes en la organización y consolidación de un sistema de salud. Se aplicó el año 2015 una guía de entrevistas a profundidad que fueron grabadas, transcritas y codificadas para identificar los principales núcleos o ideas aglutinadoras que tiene el grupo sobre las principales funciones del sistema de salud.

\section{RESULTADOS}

Se presentan las principales opiniones y núcleos representativos de las explicaciones que se identificaron en el discurso del grupo consultado, respecto a las funciones de rectoría, provisión de servicios y 
financiamiento del sistema de salud, así como de los factores generales que influyen en el sistema de salud. Lo expresado por los entrevistados se coloca en frases textuales y el código asignado a cada entrevistado entre paréntesis.

Los núcleos representativos de los factores generales políticos y económicos que influyen en el sistema de salud, identificados en el grupo consultado, tiene tres núcleos en los que organizan sus pensamientos y reflexiones, los cuales están interconectados entre sí, el primero se refiere al rol del Estado y las políticas de salud. Aquí se plantea que la implementación de un sistema de salud es un proceso social y por lo tanto político "todos los procesos sociales son materia de procesos políticos, los procesos políticos son indispensables" (E1) "sobretodo teniendo presente que la salud es considerada un derecho humano...y las políticas deben ser consecuentes con esa concepción" (E3).

Al ubicarlo en este ámbito político, el grupo ve la necesidad de acuerdos políticos, en ese sentido se reconoce la contribución del Acuerdo Nacional del año 2002 "hay un logro importante y es que sale el Acuerdo nacional, el cual establece las pautas de lo que es la política pública de hoy" (E9). Pero en general hay falta de consenso, que da poca continuidad de las políticas "Ia garantía del consenso es que si todos están de acuerdo, están moralmente obligados a seguir el acuerdo en la misma dirección" (E1).

El segundo núcleo representativo se refiere al financiamiento de políticas públicas e intereses políticos y globales, aquí el grupo considera que hay obstáculos, ya que "al final el poder está en quien tiene las finanzas" (E1); se cruzan intereses políticos e intereses globales que no permiten el flujo financiero al sistema de salud "estamos en un boom económico, hay dinero....nosotros le decíamos al Presidente Ollanta que aumente el presupuesto en salud del $2 \%$ al $8 \%$ porque existian fondos...pero después hubo un cambio total" (E3).

El tercer núcleo representativo se refiere a poca coordinación intergubernamental como una limitante, ya que "no hay articulación de visiones entre los di-

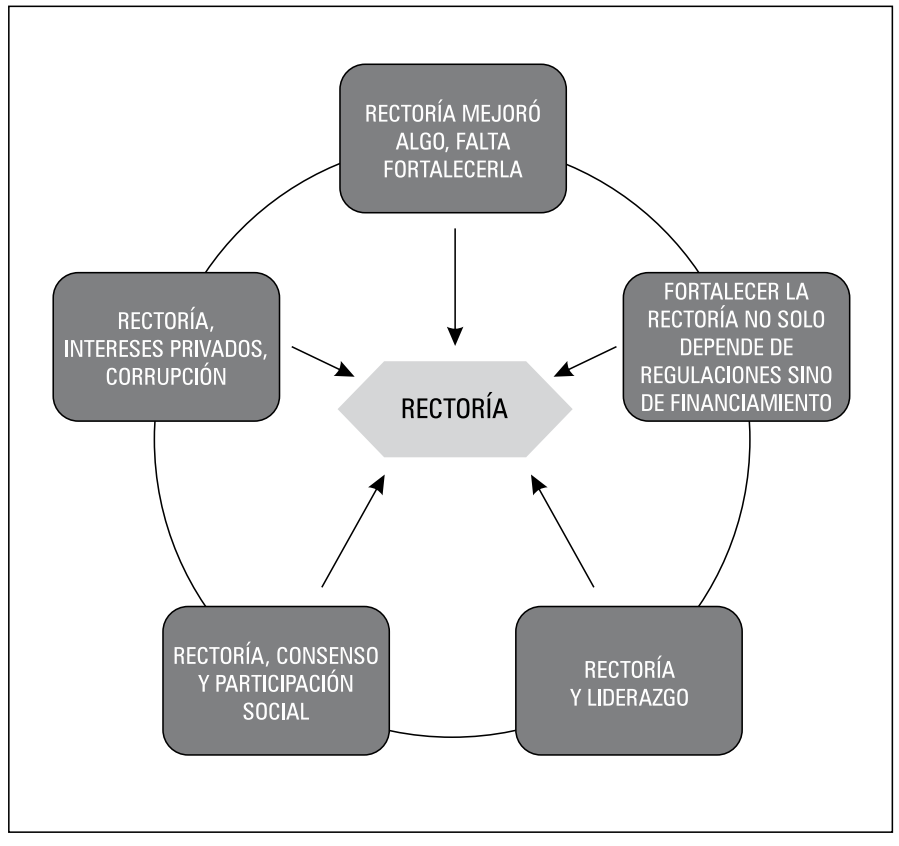

Figura 2. Representaciones sociales de la rectoria del Ministerio de Salud ${ }^{(13,16)}$

ferentes niveles de gobierno" (E4) y "la coordinación intersectorial es uno de los ejercicios más difíciles" (E10).

Respecto a la rectoría del Ministerio de salud se identificaron 5 núcleos de representaciones, las cuales están interrelacionadas como se aprecia en la figura 2.

El grupo considera que la rectoría mejoró algo pero falta fortalecerla, señalando que mejoró en base a que se han promulgado regulaciones y normativas como "la creación de la Superintendencia de salud que es un mecanismo que expresa rectoría" (E9)(12). Sin embargo, también el grupo menciona que estos avances no son significativos incluso algunos plantean que se ha debilitado: "débil rectoría ya que emite normas y nadie cumple" (E7)(E8). En todo caso "el proceso por afianzar la rectoría del MINSA es un proceso inacabado debido a que es un sistema fragmentado y muy segmentado" (E6). También se considera que la descentralización debilitó la rectoría "la descentralización afecto, los Presidentes Regionales designan a directores regionales" (E2).

El fortalecimiento de la rectoría no solo depende de regulaciones sino de financiamiento. A pesar de que se recono- ce la importancia de las normativas, estas no son suficientes "existen mecanismos pero falta ajustarlos" (E3). Pero sobre todo se requiere suficiente asignación de recursos "Ia rectoría hay que fortalecerla, todos están de acuerdo en la teoría, pero a la hora de asignar recursos ahí se amarra" (E3).

Respecto a rectoría y liderazgo para tener un solo sistema de salud, aquí se da importancia al liderazgo que debe tener el MINSA y el Ministro de Salud quien debe "hacer liderazgo, ser luchador, tiene la necesidad de concertar pero defendiendo derechos" (E6). Esta rectoría y liderazgo debe orientarse a consolidar al sistema de salud, pero con una modalidad que todavía se discute ya que unos plantean que "deberíamos tener un solo sistema de salud para el Perú, un solo sistema de salud integrado" (E3), pero hay variantes ya que "la reflexión en los últimos 10 años en el Perú ha sido: ¿es posible crear un sistema que unifique a todos? o es inevitable mantener la institucionalidad de los diferentes actores" (E6).

Respecto a rectoría, consenso y participación social, el grupo considera que la participación social en la elaboración de políticas y leyes tiene limitaciones, un ejemplo fue "el caso particular de la Ley 
30027 ...sobre alimentación saludable. Lo que pasa es que en nuestros países puedes tener leyes preciosas que a la hora de aterrizarlas tienen un reglamento....y a la hora del reglamento allí entraron las otras manos oscuras que para lo cual la sociedad civil no intervino para nada, que era el tema de las industrias y de los otros poderes" (E10), ratificado por "juzgamos por el diseño del proceso de reforma del año 2012-2013. Yo no podría decir que son escuchadas las voces en dimensión suficiente...a pesar que propuestas de la sociedad civil se han convertido luego en normas o leyes" (E12).

Sobre rectoría, intereses privados y corrupción, al grupo le preocupaba temas como la corrupción y el juego de importantes intereses económicos alrededor del sistema de salud: "hay actores fuertes alrededor de los sectores públicos que cuando ven afectados sus intereses demuestran toda su capacidad de presión; un ejemplo claro fue cuando Foro Salud en el año 2012 en una campaña por alertar sobre el tema de la publicidad de alimentos dañinos para la salud, y la forma increíblemente poderosa en la que responde la industria alimentaria a partir de voceros de líderes de opinión" (E12). Otro tema mencionado es el de la corrupción y el grave daño que le hace al sistema ya que "si hay corrupción, la plata se va para otro lado" (E3).

La descentralización que se está implementando en estos años incidió en la rectoría del Ministerio de Salud, por eso las representaciones sociales que se identificaron van en dos vías, la que considera que se implementó inadecuadamente, y la que indica que se requiere perfeccionarla para un mejor gobierno ya que "la descentralización es necesaria para el Perú porque había un centralismo asfixiante en Lima" (E3).

Respecto a que la descentralización fue implementada inadecuadamente, se plantea que "la descentralización ha dificultado todo, en este momento hay que reconocer el ministerio de salud no tiene ya ninguna injerencia en las regiones" (E1) ratificado por "la descentralización afectó, debilitó la rectoría...los Presidentes Regionales designan a directores regionales de salud" (E2).

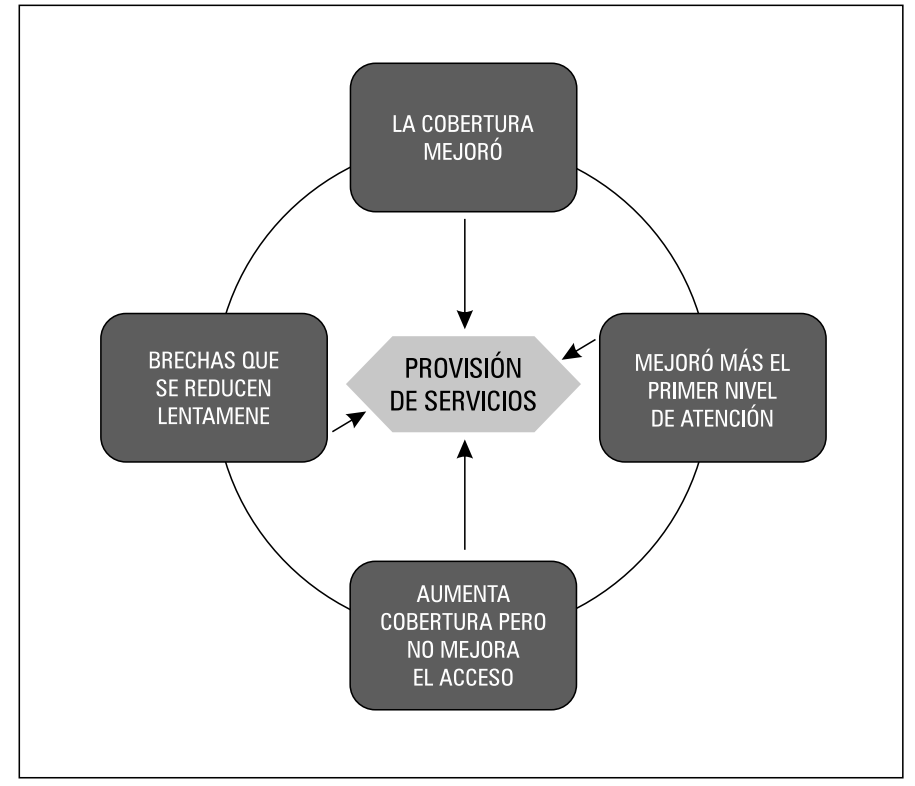

Figura 3. Núcleos representativos de provisión de servicios de salud.

Se propone que la descentralización debe mejorar pero no disminuir la rectoría del Ministerio de salud ya que "la descentralización mejora las decisiones" (E4), se debe "persistir con la descentralización pero bien regulada" (E3). Se considera que se debió preveer mecanismos para que no sea una ruptura entre las políticas nacionales y las regionales. También se plantea que el nivel central debe retomar algunas funciones: "han pasado cinco años y justamente lo que está planteando el Ministerio de Salud es cómo se recuperan las competencias" (E 13).

Los núcleos representativos de la provisión de servicios identificados en el grupo fueron cuatro, que están interconectados entre sí. Figura 3.

El grupo tiene varios núcleos de representaciones que parecerían opuestos pero no es así ya que lo que manifiestan los entrevistados son situaciones reales en un continuo entre dos extremos; así, plantean que si bien hay avances en la cobertura, no son suficientes ni han resuelto el problema del acceso para la mayoría de la población, las dos situaciones se dan: aumento de la cobertura financiera pero no del acceso a atención de salud.

Sobre que la cobertura mejoró, aquí el Sistema Integral de Salud (SIS) es reco- nocido como una de las razones para el aumento de la cobertura en base al incremento del presupuesto "el SIS ha mejorado, incrementando atención de niños, madres y adultos de la tercera edad, cubre $85 \%$ de patologías más frecuentes, el FISSAL el 20\% de patologías catastróficas" (E5) y en el último gobierno se siguió mejorando: "hay mejoras tangibles en el acceso... el Plan Esperanza es un ejemplo de ello" (E11) (E12). Sin embargo, la cobertura con seguro no logró cubrir a todos ya que el "25\% de personas no tienen nada" (E8).

Pero mejoró más el primer nivel de atención ya que el grupo valora los esfuerzos en la atención primaria: "en el diagnóstico más del primer nivel ha habido un aumento, la universalización... en situaciones de vulnerabilidad como la primera infancia, todas las gestantes, todos los niños menores de cinco años, tienen cobertura" (E11).

Se considera que aumentó la cobertura pero no mejoró el acceso, especialmente del acceso a nivel hospitalario donde hay críticas porque esto implica continuar con un modelo basado en hospitales "por más que le das presupuesto al hospital, lo único que consigues es que la demanda también aumente, o sea, le has dado mayor capacidad de oferta, 
pero has generado mayor capacidad de demanda sobre el hospital que debía haber sido trasladada al primer nivel.....eso falló (E2), "toda la gente se va a los hospitales nacionales porque el sistema está hecho para que el paciente vaya al hospital...para que consuma medicamentos... para que se gaste el dinero en la consulta, en lo curativo" (E3) y "hay que salir de los modelos puramente curativos... a los estadíos previos, que es el control de los factores de riesgo, que es la esencia de la prevención primaria" (E10). Además, en el modelo hospitalario fluyen más recursos que atraen intereses ya que "hay una tremenda inversión, bajo presión política para implementación de tecnologías que no son necesarias, son caras de mantener, poco costo-beneficio" (E2).

Las brechas se reducen lentamente y se reconoce que hay importantes brechas de infraestructura y equipamiento, de recursos humanos y de medicamentos, así se reconoce que "el Perú tiene como retos la escases de recursos, un financiamiento escaso, recursos humanos insuficientes y mala distribución del acceso a tecnologías y a medicamentos" (E12). Estas brechas explican que a pesar "que el 85\% de la población esté asegurada a su seguro público ¿significa que este $85 \%$ tenga resuelta su necesidad frente a la enfermedad? No, no es asi" (E13), "estamos en un $76 \%$ de cobertura de afiliados, pero eso no significa cobertura en prestaciones de servicios" (E5).

Pero no solo es un tema de brechas de infraestructura sino de organización, gestión y coordinación del sistema, de fortalecer el trabajo en redes de salud para resolver las necesidades de los ciudadanos. La poca articulación mantiene inequidades "ha mejorado el acceso y financiamiento pero no la oferta, falta organizar el sistema por niveles y por referencia, al hospital de especialidad neurológica le llega la migraña común" (E4).

Además, hay diferentes barreras de acceso "Yo estoy en EsSalud, aporto mensualmente; sin embargo, si voy por la vía normal a pedir una cita en traumatología la encuentro para julio, ¿que hago con mi rodilla? la barrera organizacional que te plantea el sistema para un acceso efectivo es un reto que nunca lo van a ver las autoridades porque ellos no se atienden en EsSalud" (E12).

Este déficit de infraestructura, recursos humanos y equipamiento produce exclusiones incluso para los afiliados al SIS "hay hospitales que tienen clínicas y que utilizan la infraestructura hospitalaria, los insumos hospitalarios...tu entras por aquí a la puerta del SIS y veinte metros más allá entras a la clínica pagando ochenta soles de consulta (E13).

De la mano de la brecha de infraestructura va la de recursos humanos, mencionando que hay un verdadero "drama de recursos humanos" (E8) pero también de acceso a los medicamentos "el SIS, por un modelo presupuestal público plantea una ruta larga de entregar recursos y convertirse en medicamento... no hemos tenido la capacidad de que ese dinero se convierta en tiempo real en medicamentos" (E9).

Respecto a los núcleos de representaciones sobre el financiamiento, en esta categoría las tendencias de las representaciones y reflexiones grupales van en 2 direcciones. La primera indica que la atención de salud cuesta pero no debe empobrecer. Se tiene presente el alto costo de los procedimiento diagnósticos y terapéuticos, por lo tanto estos costos deben ser asumidos por el Estado "la enfermedad para una persona es devastadora, que llegue el cáncer a un hogar y esa persona vende su casa, su carro.... por eso se plantea en la reforma del 2013 que plantea que ningún peruano debe empobrecerse en el proceso de recuperar su salud" (E7). Esta propuesta choca con la realidad de que "el Perú ha mejorado fuertemente en su financiamiento; sin embargo, no es aún ni de cerca el promedio latinoamericano" (E9)(E12). Un indicador de esto es que el gasto de bolsillo en salud se mantiene alto "el gasto de bolsillo es de 33 \% debido a que el SIS no cubre todas las necesidades y por gasto superfluo" (E6)(E13). Situación relacionada con que el ministerio de finanzas decide los recursos "yo creo que el tema de los ministerios de economía es prácticamente decisivo, como el segundo presidente del país" (E4), que se ratifica con que "Ia decisión política pasa por el presupuesto" (E3).
Se propone la creación de un solo fondo de financiamiento como una alternativa para superar la fragmentación del sistema de salud y avanzar hacia la conformación de un solo fondo que financie las prestaciones "si quisiéramos un sistema nacional de salud, debe tener un financiamiento por un lado de impuestos, impositivo, fiscal, y por otro lado también la contributiva, o sea la parte del empleador y del trabajador. Un solo fondo nacional que cubra todo" (E3).

Sin embargo, a pesar de que existen posiciones como la anterior, este es un tema que a nivel político todavía no ha sido posible "lo que se plantea con respecto a la unificación de los sistemas... en la época de Álvaro Vidal y de Alberto Tejada, se discutía bastante, hubo proyectos en el parlamento que llevaban el SIS a la Seguridad Social y otros que iban en línea contraria. Esas unificaciones que quizá hubieran podido resultar por algunas razones, pero por otras eran muy difíciles de implementar, de conseguir los acuerdos políticos para hacer eso" (E11).

Respecto a los núcleos representativos de buenas prácticas de gestión pública en salud y desafíos, la figura 4 presenta los cinco núcleos de representaciones sociales identificados y que están interconectadas entre sí, el primero se refiere a las cualidades de un Ministro de Salud, destacándose las características del ministro quien debe ser un luchador, que busque concertar, que tenga "capacidad de liderazgo, el ministro pone la agenda y tiene que tener equipo que haga labor pre frontal" (E4) (E2).

La relación con el Presidente y con el resto de ministros es clave: "es importante tener respaldo del presidente, tener equipo y la comparación internacional también ayuda" (E6) y "la interrelación directa Ministro de Salud, Ministro de Economía, y otros ministros" (E2).

El segundo se refiere a la necesidad de consolidar el sistema de financiamiento y mejorar la equidad, ya que "el fundamento para nosotros del sistema universal más allá de que haya dos o tres prestadores o tres o 4 fondos es que la respuesta pública tiene que ser igual. Un solo plan de salud para todos" (E13). 


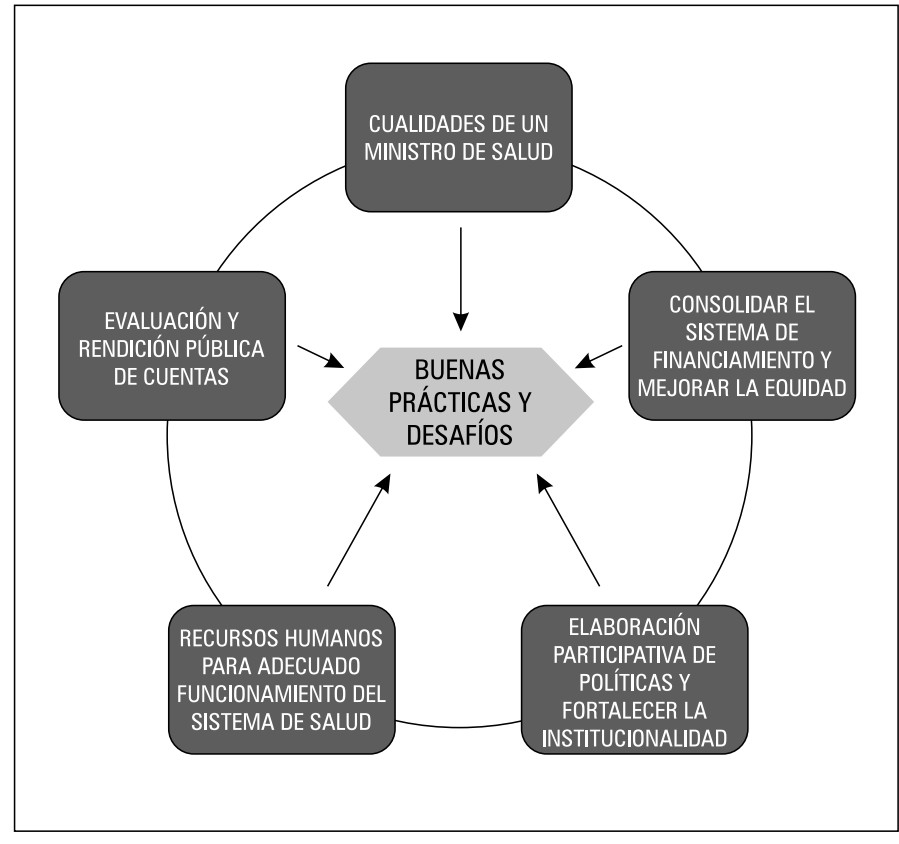

Figura 4. Núcleos de buenas prácticas de gestión en salud y desafíos.

El tercero se refiere a la construcción participativa de políticas y leyes así como fortalecer la institucionalidad "construcción participativa, incluso participación social no es te participo lo que estoy pensando hacer y después digo: esto ha sido una construcción participativa"... ¿Participativa en qué sentido? construcción participativa es: nos sentamos los diez, los diez consensuamos y ahí viene la palabra difícil "consensuar", hay que construir procesos participativos con la gente" (E2) y "las políticas deben incluir a los ciudadanos "la política debe decir: el ciudadano ¿cómo está?” (E9).

El grupo considera que hay que consolidar la institucionalidad del sistema que implica no solo fortalecer al Ministerio de Salud sino a otras instituciones como la Comisión Intergubernamental de Salud y el Consejo Nacional de Salud "el Consejo Nacional de Salud creemos que debería ser el camino para recuperar la rectoría sin dejar de lado obviamente por ejemplo la Comisión Intergubernamental de Salud" (E13).

El cuarto se refiere a contar con recursos humanos: "yo creo que la clave es la formación del personal, la preparación a todos los niveles" (E1) y "contratar profesionales de cada localidad y
capacitarlos"(E8) y mejorar la distribución del personal de salud ya que "el $83 \%$ de los especialistas están en las 3 ciudades más grandes del país" (E6).

El quinto se refiere a evaluación y rendición pública de cuentas, "si no tienes un referente con el cual contrastar 10 que haces ¿cómo te mides? Si no tienes indicadores, si no tienes metas ¿cómo te mides? te mides con lo que tú mismo dices que vas a hacer... eso no es una medición" (E2). Entonces una buena práctica a fortalecer es la evaluación del sistema y del financiamiento: "evaluar periódicamente al sistema, evaluación técni$c a^{\prime \prime}$ (E8) y el monitoreo de las políticas porque "hay un segundo quiebre entre la política y la gestión sanitaria...hay un quiebre entre lo que decide el político, el Presidente, los ministros y lo que está ocurriendo en el terreno" (E9).

El principal desafío es lograr acceso universal mediante un solo plan de salud para todos y una importante articulación entre todas las instituciones "no solo para ese $85 \%$ sino para todo el país... lo que queremos es un sistema universal... un solo plan para todos los peruanos" (E13).

Otro reto es involucrar a todos los sectores relacionados con la salud, como el caso de la universidad "yo no siento que la academia tenga un rol protagónico de propuesta diferenciada de la corriente, me puedo equivocar, yo hablo más orientado a mi ramo farmacéutico (E9). Finalmente, se visibiliza a la corrupción como un peligro para el sistema de salud y que "hay que eliminar la corrupción...el favoritismo".

\section{DISCUSIÓN}

Los resultados, que en este tipo de investigación cualitativa están enfocados a entender y explicar desde la visión y los discursos de los actores sociales permitieron tener una visión de la evolución del sistema de salud del Perú en el período estudiado. Para el análisis del sistema de salud se tuvo como referencia la metodología usada por el Instituto Suramericano de Gobierno en Salud (ISAG), en la que destaca el estudio de la rectoría, provisión de servicios de salud y el financiamiento como componentes importantes de un sistema de salud. ${ }^{(19)}$ El estudio de los sistemas de salud ${ }^{(20)}$ y sus componentes son reconocidos en la agenda internacional en salud como elementos esenciales del sistema de salud y se requiere su permanente estudio y seguimiento con el objetivo de ayudar a lograr el acceso y cobertura universal de salud. (21)

Los resultados evidencian que un adecuado funcionamiento del sistema de salud es un requisito para alcanzar la universalización del derecho a la salud, pero, siendo el sistema de salud un proceso social requiere de acuerdos políticos y sociales. Un escenario de ese tipo permitirá implementar políticas públicas de salud a largo plazo, las cuales serán un sustento para alcanzar metas sanitarias que beneficien a la población.

Otro aspecto presente es que para que las políticas de salud se desarrollen adecuadamente e impacten positivamente se necesita una buena coordinación interinstitucional entre las diferentes instancias gubernamentales. Esto en la medida que la salud de la población tiene que ver con diferentes aspecto de la vida de la población como el acceso a trabajo, acceso a educación y a un adecuado ambiente económico y político. 
Respecto a la rectoría, que es la capacidad de conducción del sistema, ${ }^{(22)}$ es vista como una de las funciones clave en el funcionamiento del sistema de salud; así, según los entrevistados, a pesar que existen hechos encaminados al fortalecimiento de la capacidad de control del sistema como es la presencia de la superintendencia de la salud (SUSALUD), todavía la rectoría continua débil, lo que se expresa en que coexisten otros sistemas como el de la seguridad social y fuerzas armadas, sumado a que las normativas del Ministerio de Salud no son cumplidas en todo el sistema de salud.

Se reconoce como importante para la conducción del sistema de salud al liderazgo y capacidad de concertación que debe tener un Ministro de Salud. La presencia de un ministro con estas cualidades permite periodos de fortalecimiento de la rectoría del Ministerio de Salud y avances de todo el sistema.

Sobre la rectoría también influyen los intereses privados, corrupción, y un inadecuado uso de fondos públicos; al grupo le preocupa temas como el juego de importantes intereses económicos alrededor del sistema de salud. Situación que también está ocurriendo en otros países sudamericanos ${ }^{(23)}$.

La descentralización que se implementó en estos años incidió en la rectoría del sistema y se requiere perfeccionarla para un mejor desempeño del sistema de salud, especialmente formando masa crítica en las regiones.

En relación a la provisión de servicios de salud se reconoce avances en la cobertura especialmente en el nivel primario de atención, pero en los niveles de mayor complejidad como hospitales el acceso ha mejorado poco debido a brechas de recursos humanos, camas hospitalarias y equipamiento. Situación que también se evidencia en otros países como Ecuador. ${ }^{(24)}$

La participación social como elemento que permite vigilancia para que las políticas y los programas de salud funcionen adecuadamente no tiene una presencia consolidada. Esta situación podría traducirse en falta de exigibilidad para que se concreten verdaderos impactos positivos en la salud de los ciudadanos. La limitada participación social en el funcionamiento del sistema de salud y en la implementación de políticas públicas en salud es reconocida como un desafío pendiente. ${ }^{(25)}$

El financiamiento del sistema de salud es un aspecto de mucha preocupación de los entrevistados, si bien en el periodo estudiado se evidencia incremento del financiamiento público y el fortaleciendo del sistema integrado de salud, todavía no existe una protección financiera adecuada cuando los ciudadanos se enferman, lo que se traduce en un alto gasto de bolsillo por parte de los pacientes y sus familiares. Este es un tema que la OMS/OPS recomienda que debe ser de alta preocupación por parte de los gobiernos nacionales. ${ }^{(26)}$

El grupo de expertos considera que la rectoría en salud ha seguido un lento fortalecimiento en estos años, se reconoce el mejoramiento de la cobertura financiera pero no del acceso a servicios de salud y hay serias brechas de recursos humanos, infraestructura, equipamiento y acceso a medicamentos, a lo que se suma ineficiencia organizacional. La conformación de un solo fondo de salud se propone como una alternativa para armonizar el funcionamiento del sistema de salud.

\section{REFERENCIAS BIBLIOGRÁFICAS}

1. Organización Mundial de la Salud. Estrategia de la OMS para los sistemas de salud .Disponible en http:// www.who.int/healthsystems/strategy/es/ Recuperado el 20 marzo 2016.

2. Organización Panamericana de la Salud. Estrategia para el acceso universal a la salud y la cobertura universal de salud. Consejo Directivo OPS. Washington. Octubre 2014.

3. Gomes Temporão, J.Sistemas universales de salud en el mundo en transformación. En: Faria M, Feo O, Giovanella L, Ruiz G, Tobar S.( Eds): Sistemas de salud en Suramérica. Rio de Janeiro: ISAGS. 2012.

4. Organización Mundial de la Salud. Qué es un Sistema de Salud? .2005. Disponible en http://www.who. int/features/qa/28/es/ Recuperado el 12 de enero 2016.

5. Faria M, Feo O, Giovanella L, Ruiz G, Tobar S.(Eds) Sistemas de Salud en América. Desafíos para la universalidad, la integralidad y la equidad (pp.21-70). Rio de Janeiro: ISAGS. 2012 . Disponible en http:// www.isags-unasur.org/uploads/biblioteca/2/bb[8] ling[2]anx[9].pdf Recuperado el 13 de enero de 2016

6. Organización Mundial de la Salud. Sistemas de salud Disponible en http://www.who.int/topics/health_systems/es/. Recuperado el 20 de marzo 2016.

7. Organización Mundial de la Salud. Diez datos acerca de la cobertura sanitaria universal. Disponible en www.who.int/features/factifiles/universal_health_coverage es/. Recuperado el 15 de abril 2016.

8. De Habich M. Seminario internacional sobre reforma del sector salud. MINSA .Lima, junio 2013.

9. Malo-Serrano M, Malo-Corral N. Reforma de salud en Ecuador: nunca más el derecho a la salud como un privilegio. Rev peru med exp salud pública. 2014;31(4):754.

10. Organización Mundial de la Salud. Cumbre ministerial sobre investigación en salud. Disponible en www. who.int/rpc/summit/es/index3.html Recuperado el 25 marzo 2016.

11. Organismo Andino de Salud, Convenio Hipólito Unanue. Creado en 1971. Sede Lima, Perú.

12. MINSA. Ley marco de aseguramiento universal en salud. Disponible en www.minsa.gob.pe/dgsp/ar Recuperado 15 enero 2016.

13. Vera M. Significado de la calidad de vida del adulto mayor. Saarbrucken, Dentschland, Alemania: Ed. Académica española. 2011:11-36.

14. Mora M. Citando a Darío Páez en La teoría de las representaciones sociales de Serge Moscovici. Atenhea digital-num.2 otoño 2002.

15. Instituto de Estudios Peruanos. La Representación Social: un concepto perdido. Disponible en cholonautas.edu.pe/modulo/upload/tallmosc.pdf. Recuperado el 15 abril 2016.

16. Ruales J, Orozco M. Módulo II. Ejes críticos del desempeño de los sistemas de salud. 2009. Disponible en http://www.paho.org/PAHO- USAID/documents/ events/nicaragua09/Sistemas_Salud_componentes_dimensiones-OPS. Recuperado el 10 Febrero de 2016.

17. Estudio del Psicoanalisis y Psicología. Serge Moscovici: representaciones sociales. Disponible en psicopsi.com/serge-moscovici-representacionessociales. Recuperado el 30 enero 2016.

18. Ponce L, Amalia R. Representaciones sociales de la enseñanza-aprendizaje de la investigación en enfermería en la Facultad de Medicina UNMSM. 2012. Tesis para optar el Grado Académico de Doctor en Enfermería. Lima - Perú. 2014.

19. ISAGS. Sistemas de salud en Suramérica: desafíos para la universalidad la integralidad y la equidad. Rio de Janeiro: ISAGS; 2012.

20. Roncarolo F, Boivin A, Denis J-L, Hebert R, Lehoux P. What do we know about the needs and challenges of health systems? A scoping review of the international literature. BMC Health Serv Res. 2017;17(1):636.

21. OPS/OMS. Resolución cd53.r14 estrategia para el acceso universal a la salud y la cobertura universal de salud. Disponible en: http://iris.paho.org/xmlui/ bitstream/handle/123456789/7652/CD53-R14-s.pdf

22. Lopez-Cevallos DF, Chi C. Assessing the context of health care utilization in Ecuador: a spatial and multilevel analysis. BMC Health Serv Res. 2010;10:64.

23. "El 30 por ciento del presupuesto en salud se malgasta" [Internet]. [citado 24 de diciembre de 2017]. Disponible en: http://www.semana.com/ amp/entrevista-a-gerente-general-de-pfizer-paracolombia-y-venezuela/550668

24. Cañizares R. El Sistema Nacional de Salud de Ecuador: Estudiarlo para mejorarlo. Alternativas. 2017;17(2):60-4

25. Malo-Serrano M, Malo-Corral N. Health reform in Ecuador: never again the right to health as a privilege. Rev Peru Med Exp Salud Publica. 2014;31(4):754-61.

26. Grupo Banco Mundial/OMS. Hacia la cobertura universal en salud y la equidad en América Latina y el Caribe. Disponible en : https://openknowledge.worldbank. org/bitstream/handle/10986/22026/9781464811777. pdf? sequence $=8 \&$ isAllowed $=y$ 